\title{
Non-Operative Management of Femoroacetabular Impingement: A Prospective Study
}

\author{
Andrew T. Pennock, MD¹, James D. Bomar, MPH², Kristina Parvanta, ATC, OPA-C ${ }^{1}$, Vidyadhar Vinayak Upasani, MD ${ }^{1}$
}

${ }^{1}$ Rady Children's Hospital, San Diego, CA, USA, ${ }^{2}$ Rady Children's Hospital, San Diego, San Diego, CA, USA.

Objectives: Little attention has been given to the non-operative management of femoroacetabular impingement (FAI) in the literature despite a rapidly expanding body of research on the topic. The purpose of the current project was to perform a prospective study utilizing a non-operative protocol on a consecutive series of patients presenting to our clinic with FAI.

Methods: Between 2013 and 2016, patients referred to our clinic for hip pain that had a positive impingement sign were prospectively recruited in a non-operative FAl study. The protocol consisted of an initial trial of rest, physical therapy, and activity modification with a focus on avoidance of high hip flexion (Activity Mod group). Patients who remained symptomatic were then treated with an image-guided intra-articular steroid injection (Injection group). Patients with residual symptoms were then offered arthroscopic treatment (Surgery group). Outcome scores were collected at 12 and 24 months. Statistical analysis was performed to identify risk factors for failure of non-operative treatment.

Results: 129 symptomatic hips in 100 patients were enrolled. After our exclusion criteria were applied, 110 hips in 84 patients remained with a mean follow-up of 25.5 months. Eighty-one hips (73.6\%) were managed with PT, rest, and activity modification alone. Thirteen hips (11.8\%) required a steroid injection, but did not progress to surgery. Sixteen hips (14.5\%) required arthroscopic management. All three groups saw similar improvements in modified Harris hip score $(m H H S)(p=0.706)$ and non-arthritic hips score (NAHS) $(p=0.712)$. Initial, and most recent, mHHS and NAHS can be found in Table 1. Labral tears were distributed similarly among the three groups $(n=41, p=0.09)$ and saw similar improvements in outcomes $(p>0.5)$ as hips without labral tears. The surgical patients attempted non-operative treatment for a mean of 8.8 months prior to surgical intervention. Delays in surgery were not associated with worse outcomes. Cam lesion size, acetabular coverage, and the presence of a labral tear were not associated with non-operative treatment failure $(p=0.579)$.

Conclusion: A large majority of adolescent patients presenting with FAl can be managed nonoperatively with significant improvements in outcomes scores and continuation of sport at a mean follow up of two years. This is the first prospective study evaluating the outcomes of a standardized non-operative protocol for the management of FAI. Our results show that a commitment to non-operative care can work for a large percentage of patients. We will be following these patients further into the future to examine the durability of these results.

\begin{tabular}{|c|c|c|c|c|}
\hline & \multicolumn{2}{|c|}{ Modified Harris Hip Score } & \multicolumn{2}{|c|}{ Non-Arthritic Hip Score } \\
\hline & Initial & Most Recent & Initial & Most Recent \\
\hline Activity Mod & $70.0 \pm 13.3$ & $89.8 \pm 12.2$ & $74.2 \pm 16.4$ & $87.1 \pm 14.8$ \\
\hline Injection & $67.9 \pm 11.2$ & $91.3 \pm 9.2$ & $72.2 \pm 13.1$ & $86.1 \pm 10.2$ \\
\hline Scope & $67.6 \pm 9.3$ & $87.9 \pm 11.4$ & $72.7 \pm 11.3$ & $89.2 \pm 9.3$ \\
\hline p-value & 0.748 & 0.676 & 0.699 & 0.433 \\
\hline
\end{tabular}

The Orthopaedic Journal of Sports Medicine, 6(7)(suppl 4)

DOI: $10.1177 / 2325967118$ S00069

(C)The Author(s) 2018

This open-access article is published and distributed under the Creative Commons Attribution - NonCommercial - No Derivatives License (http://creativecommons.org/licenses/by-nc-nd/4.0/), which permits the noncommercial use, distribution, and reproduction of the article in any medium, provided the original author and source are credited. You may not alter, transform, or build upon this article without the permission of the Author(s). For article reuse guidelines, please visit SAGE's website at http://www.sagepub.com/journals-permissions. 